INPLASY

PROTOCOL

To cite: Hsu et al. Efficacy of Memantine for the Depressive Symptoms in Major Mental Diseases: A Systematic Review and Updated Metaanalysis of Randomized Controlled Trials. Inplasy protocol 202210080. doi: 10.37766/inplasy2022.1.0080

Received: 16 January 2022

Published: 16 January 2022

Corresponding author:

Tien-Wei Hsu

twhsu@vghks.gov.tw

Author Affiliation:

Department of Psychiatry, Kaohsiung Veterans General Hospital, Kaohsiung, Taiwan.

Support: None.

Review Stage at time of this submission: The review has not yet started.

Conflicts of interest:

None declared.

\section{Efficacy of Memantine for the Depressive Symptoms in Major Mental Diseases: A Systematic Review and Updated Meta-analysis of Randomized Controlled Trials}

Hsu, TW1; Chu, CS2; Liang, CS 3 .

Review question / Objective: The aim is to assess the effectiveness of memantine on depressive symptoms of patients with major psychiatric diseases.

Condition being studied: Memantine, an anti-dementia drug, as an NMDA-antagonist, has been reported neuroprotective effects. Another NMDA-antagonist, ketamine has been used in treatment-resistant major depression now.

Information sources: We will search the EMBASE, The Cochrane Library, PubMed, MEDLINE from the date of inception.

INPLASY registration number: This protocol was registered with the International Platform of Registered Systematic Review and Meta-Analysis Protocols (INPLASY) on 16 January 2022 and was last updated on 16 January 2022 (registration number INPLASY202210080).

\section{INTRODUCTION}

Review question / Objective: The aim is to assess the effectiveness of memantine on depressive symptoms of patients with major psychiatric diseases

Condition being studied: Memantine, an anti-dementia drug, as an NMDAantagonist, has been reported neuroprotective effects. Another NMDAantagonist, ketamine has been used in treatment-resistant major depression now.

\section{METHODS}

Search strategy: We will search the EMBASE, The Cochrane Library, PubMed, MEDLINE from the date of inception. key word: (Memantine OR NMDA antagonist 
OR NMDA blocker OR N-methyl Daspartate antagonist OR NMDA blocker) AND (depression OR depressive symptom OR major depression OR bipolar OR unipolar OR mood disorder OR schizophrenia OR schizoaffective disorder) Mesh term: Bipolar disorder, Major depression, Schizophrenia, Memantine.

Participant or population: Patients with schizophrenia or bipolar disorder, or major depressive disorder.

Intervention: Memantine.

Comparator: Placebo control.

Study designs to be included: Placebocontrolled RCT only.

Eligibility criteria: 1. studies in English 2. placebo-controlled RCT 3. Reported depressive inventory 4 . Sensitivity test: subgroup analysis and meta-regression.

Information sources: We will search the EMBASE, The Cochrane Library, PubMed, MEDLINE from the date of inception.

Main outcome(s): Depressive score, including HAM-D, MARDS, BDI, CSDD.

Additional outcome(s): The response rate, the remission rate.

Quality assessment / Risk of bias analysis: We employed risk of bias 2.0 from Cochrane.

Strategy of data synthesis: When different scales were used between studies, standardized mean differences (SMD) between treatment groups were calculated for each trial and used to derive a total estimate of treatment effect on the outcomes. The SMD is a summary statistic in a meta-analysis when the studies assess the same outcome but with different scales. For interpretation of effect sizes, we followed the rules of classifying 0.8 as large.

Subgroup analysis: Different diagnosis.
Sensitivity analysis: 1. Subgroup analysis 2. Meta-regression.

Language: English.

Country(ies) involved: Taiwan.

Keywords: memantine; major depressive disorder; schizophrenia; bipolar disorder; depressive symptoms.

Contributions of each author:

Author 1 - Tien-Wei Hsu.

Email: twhsu@vghks.gov.tw

Author 2 - Che-Sheng Chu.

Email: youngtzuchi@hotmail.com

Author 3 - Chih-Sung Liang.

Email: Icsyfw@gmail.com 